\title{
A new model for the lower overshoot layer in the Sun
}

\author{
D. Marik and K. Petrovay
}

\author{
Eötvös University, Department of Astronomy, Budapest, Pf. 32, 1518 Hungary \\ SPARC, Department of Applied Mathematics, University of Sheffield, Hicks Building, Hounsfield Road, S3 7RH, Sheffield, \\ UK
}

Received 31 January 2002 / Accepted 5 September 2002

\begin{abstract}
We present a model for the lower overshoot layer of the Sun, based on the realistic solar stratification, without the use of a "mixing-length" parameter, by solving the system of Reynolds momentum equations using the closure formalism of Canuto \& Dubovikov $(1997,1998)$. A fixed value of velocity anisotropy is assumed, and the local convection model is assumed to be valid for the convectively unstable layer. In accordance with seismic constraints, overshoot (defined as the amount by which the convectively mixed zone extends beyond its boundary in local theory) is found to be as low as about 6 percent of the pressure scale height, and it is not bounded by a discontinuity from below.
\end{abstract}

Key words. Sun: interior - methods: numerical - turbulence

\section{Introduction}

The layers situated below the convective zone of the Sun are currently considered to play an important role in the dynamo mechanism that maintains the general solar magnetic field (Petrovay 2000). Besides, turbulent mixing processes taking place in this layer are invoked to explain the lithium deficit of the solar atmosphere and to explain the remaining small discrepancies between the seismic and standard solar models (Gough et al. 1996). This turbulence may result partly from convective overshoot, and partly from rotational instabilities in the solar tachocline. The relative importance of these two sources of turbulence is an important question.

The first models for the lower overshoot layer were based on nonlocal extensions of mixing-length theory (van Ballegooijen 1982; Skaley \& Stix 1991; Zahn 1991; Umezu 1992). These predicted ${ }^{1}$ that the thermal stratification of the overshoot layer is nearly adiabatic, its extent is a significant fraction of the pressure scale height $H_{\mathrm{P}}$, and it is bounded by a discontinuity in the superadiabaticity $\Delta \nabla=\nabla-\nabla_{\text {ad }}$ from below. $\left(\nabla=\mathrm{d} \ln T / \mathrm{d} \ln P\right.$, and $\nabla_{\text {ad }}=(\partial \ln T / \partial \ln P)_{S}$, as usual, $P, T$ and $S$ being pressure, temperature and entropy.)

Such a discontinuity should, however, lead to a noticeable increase in the amplitude of the oscillatory signal in the frequency distribution of the solar oscillations (ChristensenDalsgaard 1996). Attempts to detect this have remained

Send offprint requests to: D. Marik,

e-mail: d.marik@astro.elte.hu

1 In fact, adiabatic stratification is explicitly assumed in some nonlocal MLT models, while in others it is a direct consequence of the assumption of a perfect correlation between vertical velocity and temperature fluctuations. unsuccessful (Monteiro et al. 1994; Christensen-Dalsgaard et al. 1995; Basu \& Antia 2001), which implies that the nonlocal mixing-length models are incorrect. Thus, either the overshoot layer is not bounded by a discontinuity from below, or its full extent is very small, according to Basu (1997) not more than $5 \%$ of $H_{\mathrm{P}}$. It should be stressed, however, that even in the case of a discontinuity, this limit is only valid for overshoot in the helioseismic sense, i.e. for the amount by which convection extends beyond its boundary calculated in a local model.

Note also that, as Canuto (1999) pointed out, from the point of view of stellar evolution the kinematical or dynamical extent of the overshoot, i.e. the extent of the mixed layer, is a more important quantity than its thermal extent, even if the latter were to be adiabatic. Thus throughout this paper we will define the lower boundary of the overshoot layer at the point where the kinetic energy is reduced by two orders magnitude compared to its value at $\Delta \nabla=0$.

A number of numerical simulations have also been carried out to study the problem, but they have not been conclusive. Owing to the extremely different dynamical and thermal timescales in the solar interior, the simulations are either very far from the real solar interior in parameter space (Roxburgh \& Simmons 1993; Hurlburt et al. 1994; Singh et al. 1995; Saikia et al. 2000), or they never reach a thermally relaxed state (Andersen 1994; Freytag et al. 1996).

In these circumstances, the most promising way to model the overshooting layer is the Reynolds stress method, based on the well known Reynolds momentum hierarchy of the hydrodynamical equations for a turbulent medium. Our aim in such a model is to determine the radial distribution of the turbulent kinetic energy $k$, the mean square relative temperature fluctuation $q$, the normalized energy flux $J$ and the energy dissipation 
rate $\varepsilon$ under the convectively unstable region. Note that a determination of $\varepsilon$ is equivalent to the determination of the mixing length $l$ as this parameter is usually introduced by defining $l=k^{3 / 2} / \varepsilon$. Thus, in this approach the mixing length is determined consistently within the model and it is not treated as a free parameter.

The Reynolds moment equations for $k, q$ and $J$ were solved for the lower overshoot layer by Unno \& Kondo (1989), Xiong \& Chen (1992), and Xiong \& Deng (2001), resulting in significant non-adiabatic overshoot. However, in those calculations $l$ was treated as a free parameter, arbitrarily set to be equal to $H_{\mathrm{P}}$. The use of an equation for $\varepsilon$ to get rid of the free parameter was suggested by Canuto (1993) and it has been applied in a simplified $k-\varepsilon$ model by Petrovay (1998). A fully consistent Reynolds stress formalism for stellar convection has been developed in a series of papers by Canuto and co-workers. In this paper we will employ a formalism basically identical to that of Canuto \& Dubovikov $(1997,1998)$. This formalism has been shown to agree well with numerical simulation results for a generic convective flow with inefficient convection and unit Prandtl number (Kupka 1999).

By solving the momentum equations for the realistic solar stratification, here we present the first model of the lower overshoot layer without an assumed mixing length parameter.

\section{Equations and closure}

We will use the following notations. Any variable $f$ is split into a mean and a fluctuating part as $f=\bar{f}+f^{\prime}$. The velocity $\boldsymbol{v}$ has only a fluctuating part. We assume plane parallel geometry and the depth $z$ is measured from $\Delta \nabla=0$, so the gravity acceleration $g$ is positive. Beside $\varrho$ for density, we also introduce the notations $q=\overline{\left(T^{\prime} / T\right)^{2}}, J=\overline{w\left(T^{\prime} / T\right)}, w=v_{z}, k=\overline{v^{2}} / 2=\overline{w^{2}} / f_{\mathrm{a}}$ where $f_{\mathrm{a}}$ is an anisotropy parameter.

We make the following assumptions:

$-\varrho^{\prime} / \varrho \ll 1 ;$

- the Reynolds number $R e \gg 1$, while the Prandtl number $v / \chi \ll 1$

- the extent of the overshoot layer $d \ll H_{\mathrm{P}}$, where $H_{\mathrm{P}}$ is the pressure scale height;

- the turbulent flow field is characterized by a mild and fixed value of the anisotropy $f_{\mathrm{a}} \in O(1)$. This assumption is made for simplicity only.

With these assumptions we can use the Boussinesq approximation $\varrho^{\prime} / \varrho=-\delta_{\mathrm{P}}\left(T^{\prime} / T\right)$ where $\delta_{\mathrm{P}}$ is an order of unity factor ( $\delta_{\mathrm{P}}=1$ for full ionization). Following standard practice, we will also neglect the $\overline{\boldsymbol{v} \nabla P^{\prime}}$ term in the equation for $k$.

The detailed derivation of the Reynolds momentum equations was given by many authors (Xiong 1980; Canuto 1993; Grossman et al. 1993). With the assumptions and notations mentioned above, and after customary dimensional modelling of some terms, they read

$\partial_{t} k=-\partial_{z} F_{\mathrm{k}}+\delta_{p} g J-\varepsilon$

$\partial_{t} J=-\partial_{z} F_{J}-C_{q J} \delta_{p} g q+C_{k J} f_{\mathrm{a}} \frac{\Delta \nabla}{H_{\mathrm{P}}} k-\frac{J}{\tau_{p \theta}}+\frac{1}{2} \frac{\chi}{T} \partial_{z}^{2}(J T)$

$$
\begin{aligned}
& \partial_{t} q=-\partial_{z} F_{q}+2 \frac{\Delta \nabla}{H_{\mathrm{P}}} J-\frac{q}{\tau_{p}}+\frac{1}{2} \frac{\chi}{T^{2}} \partial_{z}^{2}\left(q T^{2}\right) \\
& \partial_{t} \varepsilon=-\partial_{z} F_{\varepsilon}+C_{J_{\varepsilon}} \delta_{p} g \frac{\varepsilon}{k} J-C_{\varepsilon} \frac{\varepsilon^{2}}{k}
\end{aligned}
$$

where the non-local fluxes are

$F_{\mathrm{k}}=\overline{w v^{2} / 2}, \quad F_{q}=\overline{w\left(T^{\prime} / T\right)^{2}}$

$F_{J}=\overline{w^{2} T^{\prime} / T}, F_{\varepsilon}=\overline{w \varepsilon_{1}}$

$\varepsilon_{1}$ being the local dissipation rate. For the coefficients we use the following values: $C_{k J}=1.0, C_{q J}=1, f_{\mathrm{a}}=1, C_{\varepsilon}=1.92$, $C_{J \varepsilon}=1.44, \delta_{\mathrm{P}}=1$.

These equations are essentially a special case of the equations of Canuto \& Dubovikov $(1997,1998)$ for a thin layer, and, for simplicity, with fixed anisotropy. (Note, however, that our definiton of $J$ and $q$ slightly differs from theirs.) Correspondingly, we supplement Eqs. (1)-(4) with the closure suggested by Canuto \& Dubovikov (1997):

$\partial_{z} F_{\mathrm{k}}=-\frac{1}{3} \frac{\partial}{\partial z}\left[v_{t} \Delta^{-1} \frac{\partial}{\partial z}(K \Delta)\right]$,

$\partial_{z} F_{J^{\prime}}=-\frac{1}{3} \frac{\partial}{\partial z}\left[v_{t} \Delta^{-1} \frac{\partial}{\partial z}\left(\overline{w T^{\prime}} \Delta\right)\right]$

$\partial_{z} F_{1 / 2 T^{\prime 2}}=-\frac{35}{33} \chi^{-1} \frac{\partial}{\partial z}\left[v_{t}^{2} \Delta^{-2} \frac{\partial}{\partial z}\left(\frac{1}{2} \overline{T^{\prime 2}} \Delta^{2}\right)\right]$,

$\partial_{z} F_{\varepsilon}=-\frac{1}{2} \frac{\partial}{\partial z}\left[v_{t}\left(1+\sigma_{t}^{-1}\right) \frac{\partial \varepsilon}{\partial z}\right]$

where $\Delta=K^{2} \varepsilon^{-4 / 3}, v_{t}=\frac{2}{25} K^{2} / \varepsilon, \sigma_{t}=0.72$ and $\chi$ is the radiative conductivity. Since $\overline{w T^{\prime}}=J T$ and $\overline{T^{\prime 2}}=q T^{2}$, we can obtain $\partial_{z} F_{J}$ and $\partial_{z} F_{q}$ multiplying $\partial_{z} F_{J^{\prime}}$ by $1 / T$ and $\partial_{z} F_{1 / 2 T^{\prime 2}}$ by $2 / T^{2}$.

Finally, the timescales $\tau_{p \theta}$ and $\tau_{p}$ were calculated using formulae (34) of Canuto \& Dubovikov (1998).

\section{Numerical solution}

We are looking for stationary solutions of Eqs. (1)-(4), in which case the system reduces to an eighth-order system of ordinary differential equations. The condition of flux equilibrium

$F_{\mathrm{c}}+F_{\mathrm{k}}+F_{\mathrm{r}}=F_{\odot}=$ const.

where $F_{\odot}$ is the total solar flux, $F_{\mathrm{c}}$ is the convective heat flux, $F_{\mathrm{k}}$ is the kinetic energy flux, and $F_{\mathrm{r}}$ is the radiative flux, must also be coupled to the problem.

The upper boundary conditions are set in a point slightly within the convectively unstable layer, where we fit the solution to the solution given by a local convection theory. The equations of local theory are identical to Eqs. (1)-(4), except that the non-local fluxes are not present and the radiative terms are negligible. So these equations reduce to an algebraic system for our variables. As the system is redundant, in addition we also use the condition (10) to set the boundary values. The only free parameter is $\Delta \nabla$ which was given a small positive value $\left(\sim 10^{-8}\right)$. 
A relaxation method was used to solve this nonlinear, coupled system of differential equations. We start the integration from an arbitrary initial distribution where the dependent variables are Gaussian functions of $z$, of a width comparable to $H_{\mathrm{P}}$. During integration the following restrictions were imposed:

- Below the point where $k=0$ or $\varepsilon=0$, all dependent variables are set to zero.

- Wherever division by $k$ or $\varepsilon$ occurs in the equations we have used the condition $k \geq 10^{-100}$ and $\varepsilon \geq 10^{-100}$, to avoid floating point overflow.

- At points where the velocity-temperature correlation $\hat{J} \equiv$ $J /\left(f_{\mathrm{a}} k q\right)^{1 / 2}>1$ or $<-1$, this quantity is set to 1 or -1 , respectively. Note that this only affects the (physically irrelevant) intermediate stages during relaxation, while in the final converged solution, the unphysical situation mentioned above does not occur.

In the initial state we use a linear profile for $\Delta \nabla(\Delta \nabla=$ $\left.-0.1 z / H_{\mathrm{p}}\right)$; this crudely represents the actual run of the thermal stratification below the convectively unstable layer. The iteration goes by making one "timestep" using the full, timedependent set of Eqs. (1)-(4), followed by an instant adaptation of the temperature profile to ensure the condition of the constancy of the solar flux. This latter is achieved by calculating the kinetic flux $\left(F_{\mathrm{k}}=1 / 2 \varrho{\overline{w^{2}}}^{3 / 2}\right)$ and the convective flux $\left(F_{\mathrm{c}}=C_{\mathrm{p}} \varrho \overline{w T^{\prime}}\right)$, and subtracting them from the known solar flux to get the radiative flux

$F_{\mathrm{r}}=\frac{4 \sigma}{3 \varrho \kappa} \frac{\mathrm{d} T^{4}}{\mathrm{~d} r}$

from which we can calculate a new temperature stratification ( $\kappa$ is the opacity and $\sigma$ is the Stefan-Boltzmann constant).

The whole process is repeated until it converges to a stationary state. The relaxation does not describe a real temporal evolution, as the instant adaptation of the temperature profile is unphysical, but in this manner we can circumvent the problem of forbiddingly large radiative/dynamical timescale ratios. Thus, the technique is essentially an artificially accelerated thermal relaxation.

As the layer is thin, the perturbation of the hydrostatic equilibrium is expected to remain small. The background values of $g(z), \rho(z), \kappa(z)$ and $H_{\mathrm{P}}(z)$ were thus assumed to be unaffected by overshooting, and they were taken from a more recent version of the solar model of Guenther et al. (1992).

\section{Results and discussion}

Our stationary solution is presented in Figs. 1 and 2. The turbulent length and time scales in Fig. 2 are defined as $l=k^{3 / 2} / \varepsilon$ and $\tau=k / \varepsilon$. The thickness of the overshoot layer is strikingly small, below six percent of $H_{\mathrm{P}}$, and the $\Delta \nabla$ curve does not show a particularly sharp discontinuity. This is compatible with the available helioseismic evidence (Basu \& Antia 2001). It must be stressed that, by design, our models can only yield the extent of overshoot in the helioseismic sense (i.e. the amount by which the convective zone extends beyond its boundary in local theory). It is quite possible that a proper nonlocal treatment

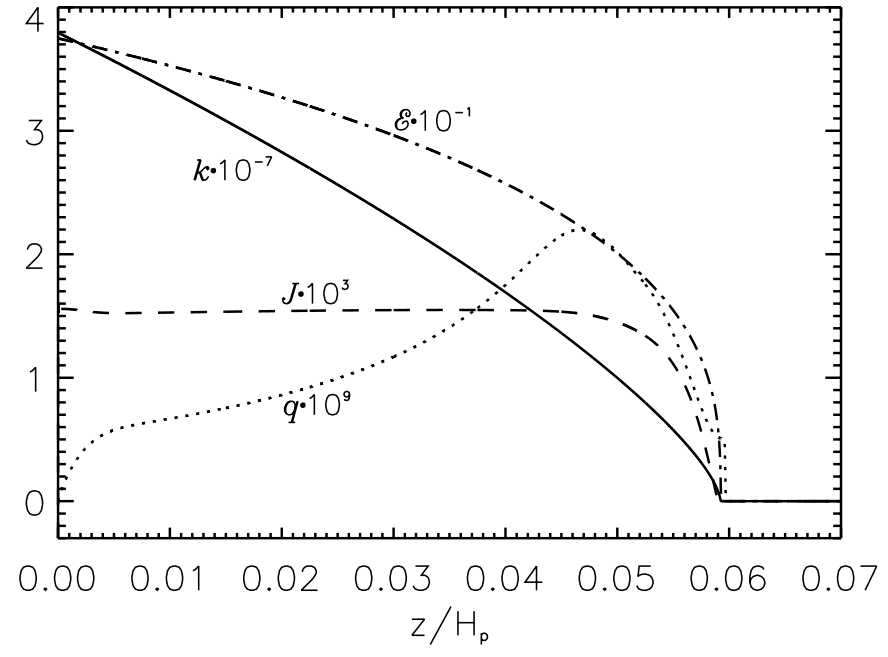

Fig. 1. Distribution of turbulent kinetic energy $k$, relative squared temperature fluctuation $q$, convective flux equivalent speed $J$, and energy dissipation rate $\varepsilon$ in our model, as functions of depth below the convectively unstable layer. (All dimensional variables here and in other figures are given in CGS units.)

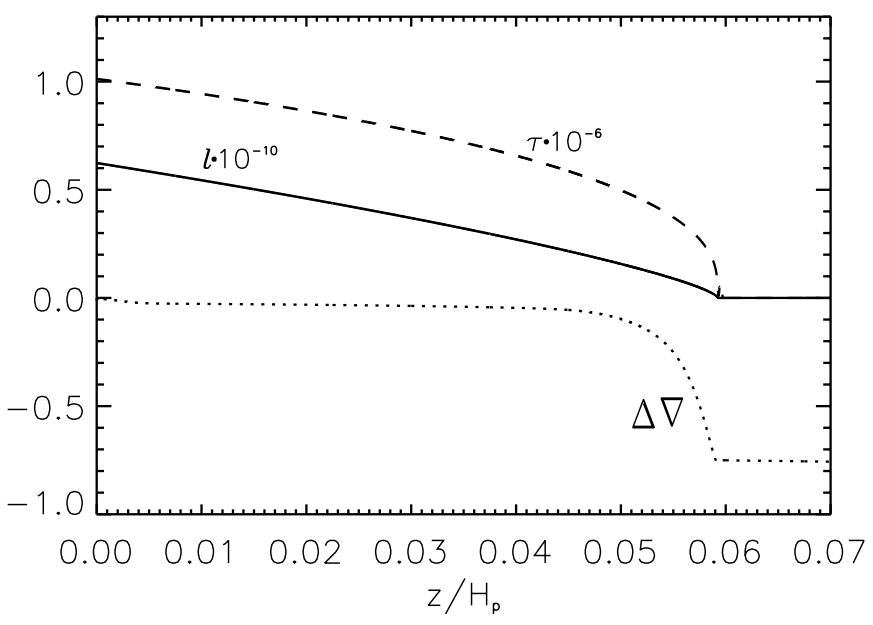

Fig. 2. Distribution of the turbulent length scale ("mixing length") $l$, time scale $\tau$, and superadiabaticity $\Delta \nabla$ in our model, as functions of depth below the convectively unstable layer.

of the full convective zone would lead to slightly subadiabatic stratification and to a downward directed $F_{\mathrm{c}}$ (the more conventional criteria for overshoot) already well above the boundary of the convective zone as defined in local theory. In this sense, the total extent of the overshoot layer may far exceed our estimate of the seismic overshoot.

It is interesting to note that the "mixing length" $l$ is found to decrease continuously towards the bottom of the overshoot layer. This underlines the incorrectness of the assumption $l=$ const. in those models that use a free length parameter.

A doubt may arise regarding the validity of these findings, given that our model only extends to the overshoot layer itself, assuming the validity of the local description for the unstable layers above. One sign of the artificiality of this sudden introduction of nonlocality is the sharp jump in the $J$ curve from negative to positive values right after $z=0$ 


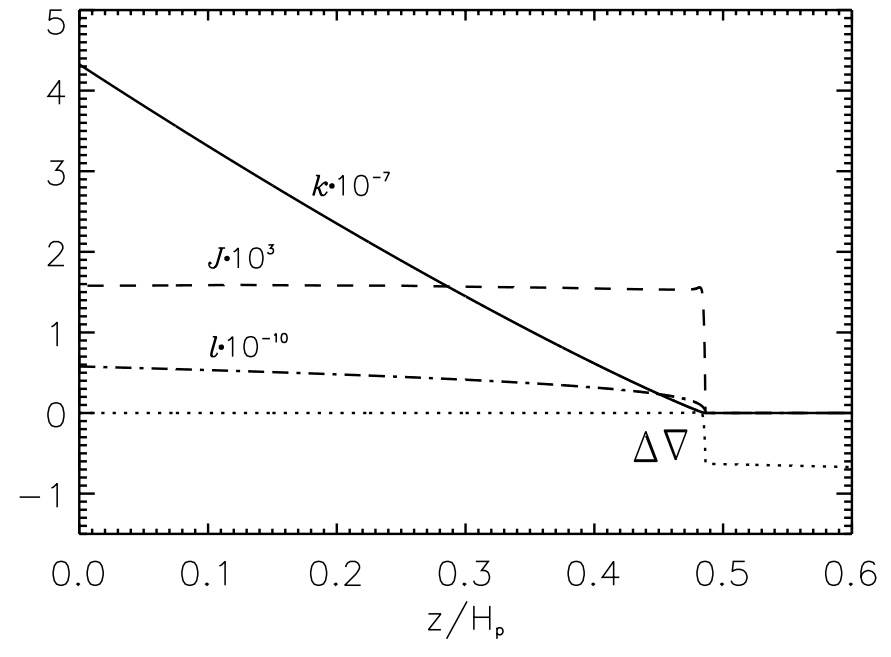

Fig. 3. Distribution of turbulent kinetic energy $k$, convective flux equivalent speed $J$, turbulent length scale $l$, and superadiabaticity $\Delta \nabla$ in an (unrealistic) solution computed with a simple downgradient closure, for comparison.

(not visible in the figure). This "patching" of our nonlocal overshoot model to the model of the SCZ computed with a local convection theory also implies that e.g. the full extent of the convective zone cannot be determined independently from our model. Indeed, placing the top boundary of the computational domain to a higher level $\left(z=-0.2 H_{\mathrm{P}}\right)$ we found that overshoot in this case started right at the upper boundary again. The total extent of this "seismic" overshoot, however, showed only moderate sensitivity to the placement of the upper boundary (resulting in an overshoot distance of $0.06 H_{\mathrm{P}}$ in the case quoted above). A model incorporating the full convective zone, with a nonlocal treatment throughout, is thus clearly a logical future extension of the present model. However, the solution presented in Fig. 3 may give us a reassurance that the small overshoot is not an artefact of our method. In this plot the stationary solution of Eqs. (1)-(4) is shown, obtained using the simple down-gradient closure

$$
\begin{aligned}
F_{k}=-\left(k^{2} / \varepsilon \partial_{z} K\right) & F_{J}=-\left(k^{2} / \varepsilon \partial_{z} J\right) \\
F_{q}=-\left(k^{2} / \varepsilon \partial_{z} q\right) & F_{\varepsilon}=-\left(k^{2} / \varepsilon \partial_{z} \varepsilon\right)
\end{aligned}
$$

instead of Eqs. (6)-(9). Note that this closure is known to be wrong, and it is only used here for comparison. It is evident that a deep overshoot can also be achieved by our method of solution; thus, the nature of the closure is the chief responsible for the smallness of the overshoot.

Yet we do not claim that our model is the final word concerning overshoot in the Sun, as several simplifiying assumptions were made both in the model and in its numerical implementation. In fact, a definitely better closure for the non-local fluxes is already available (Canuto 2001), and it has been applied to A stars successfully (Kupka \& Montgomery 2002). Further work is also needed to include a consistent treatment of the anisotropy (cf. Petrovay 1992), to clarify the dependence of the results on parameter values and closure assumptions, and to extend the domain of calculation to the unstable layer.

Acknowledgements. We are grateful to Vittorio Canuto for enlightening discussions and advice. This work was funded by the OTKA under grants No. T032462 and T034998.

\section{References}

Andersen, B. N. 1994, Sol. Phys., 152, 241

Basu, S. 1997, MNRAS, 288, 572

Basu, S., \& Antia, H. M. 2001, MNRAS, 324, 498

Canuto, V. M. 1993, ApJ, 416, 331

Canuto, V. M. 1999, ApJ, 518, L119

Canuto, V. M. 2001, J. Atm. Sci., 58, 1169

Canuto, V. M., \& Dubovikov, M. 1997, ApJ, 484, L161

Canuto, V. M., \& Dubovikov, M. 1998, ApJ, 493, 834

Christensen-Dalsgaard, J. 1996, in The Structure of the Sun, Proc. 6th Canary islands Winter School of Astrophysics, ed. T. Roca Cortés, \& F. Sánchez (Cambridge: Cambridge UP), 47

Christensen-Dalsgaard, J., Monteiro, M. J. P. F. G., \& Thompson, M. J. 1995, MNRAS, 276, 283

Freytag, B., Ludwig, H.-G., \& Steffen, M. 1996, A\&A, 313, 497

Gough, D. O., Kosovichev, A. G., Toomre, J., et al. 1996, Science, 272, 1296

Grossman, S. A., Narayan, R., \& Arnett, D. 1993, ApJ, 407, 284

Guenther, D. B., Demarque, P., Kim, Y.-C., \& Pinsonneault, M. H. 1992, ApJ, 387, 372

Hurlburt, N. E., Toomre, J., Massaguer, J. M., \& Zahn, J.-P. 1994, ApJ, 421,245

Kupka, F. 1999, ApJ, 526, L45

Kupka, F., \& Montgomery, M. H. 2002, MNRAS, 330, L6

Monteiro, M. J. P. F. G., Christensen-Dalsgaard, J., \& Thompson, M. J. 1994, A\&A, 283, 247

Petrovay, K. 1992, Geophys. Astrophys. Fluid Dyn., 65, 183

Petrovay, K. 1998, in New Eyes to See Inside the Sun and Stars, IAU Symp., 185, 121

Petrovay, K. 2000, in The Solar Cycle and Terrestrial Climate (ESA Publ. SP-463), 3

Roxburgh, L. W., \& Simmons, J. 1993, A\&A, 277, 93

Saikia, E., Singh, H. P., Chan, K. L., Roxburgh, I. W., \& Srivastava, M. P. 2000, ApJ, 529, 402

Singh, H. P., Roxburgh, I. W., \& Chan, K. L. 1995, A\&A, 295, 703

Skaley, D., \& Stix, M. 1991, A\&A, 241, 227

Umezu, M. 1992, MNRAS, 258, 107

Unno, W., \& Kondo, M. 1989, PASJ, 41, 197

van Ballegooijen, A. A. 1982, A\&A, 113, 99

Xiong, D. R. 1980, Chinese Astron. Astrophys., 4, 234

Xiong, D. R., \& Chen, Q. L. 1992, A\&A, 254, 362

Xiong, D. R., \& Deng, L. 2001, MNRAS, 327, 1137

Zahn, J.-P. 1991, A\&A, 252, 179 